\title{
Sequence-based characterization of the eight SLA loci in Korean native pigs
}

\author{
Y. J. Lee, ${ }^{*}$ K. H. Cho, † M. J. Kim,† D. M. Smith,‡ C. S. Ho,‡ K. C. Jung, ${ }^{*}$ D. I. Jin, ${ }^{*}$ C. S. Park, \\ J. T. Jeon§ \& J. H. Lee*
}

\section{Summary}

Eight swine leucocyte antigen (SLA) gene (SLA-1, SLA-2, SLA-3, SLA-6, DRA, DRB1, DQA, DQB1) alleles were identified using sequence-based typing method in three Korean native pigs used for breeding at the National Institute of Animal Science in Korea. Six new alleles in class I genes and three new alleles in class II genes have been identified in this breed and can give valuable information for xenotransplantation and disease resistance.

The major histocompatibility complex (MHC) antigens in pig are called the swine leucocyte antigens (SLA) and they can be divided into class I (SLA-1, -2, -3) and class II (SLADR, -DQ) antigens based on their structure and function (Smith et al., 2005a,b). These SLA genes are highly polymorphic and play very important roles in the immune response to infectious diseases. Many studies have associated SLA types with disease resistance and various economic traits (Gautschi \& Gaillard, 1990; Mallard et al., 1991). Recent studies have also focused on using pigs as a possible source of organ grafts for human, which makes the study of SLA antigens important for overcoming this potential immunological barrier in xenotransplantation (Xu et al., 1999; Ramachandran et al., 2004). We are interested in developing a resource herd of SLA-defined pigs for biomedical (and agriculture) research as well as a potential source of xenograft donors using the Korean native pigs. In this study, we have characterized three SLA class Ia genes (SLA-1, SLA-2, SLA-3), one SLA class Ib gene (SLA-6) and four SLA class II genes (DRA, DRB1, DQA, DQB1) in three Korean native pig boars, which have been used for breeding at the National Institute of Animal Science in Korea.

\footnotetext{
* Division of Animal Science and Resources, Research Center for Transgenic Cloned Pigs, Chungnam National University, Daejeon, South Korea, † National Institute of Animal Science, RDA, Seonghwan, South Korea, $\neq$ Department of Pathology, University of Michigan, Ann Arbor, MI, USA, and § Division of Applied Life Science, Gyeongsang National University, Jinju, South Korea
}

Received 3 March 2008; revised 3 March 2008; accepted 14 April 2008

Correspondence: J. H. Lee, Division of Animal Science and Resources, Research Center for Transgenic Cloned Pigs, Chungnam National University, Daejeon 305-764, South Korea. Tel: +82 42821 5771; Fax: +82 42825 9754; E-mail: junheon@cnu.ac.kr
Sequence-based typing (SBT) was performed by reverse transcription-polymerase chain reaction (RT-PCR) followed by cloning and sequencing of multiple clones for each SLA gene as described previously (Lee et al., 2005). Briefly, RNA was extracted from each blood sample using Trizol reagent (Gibco/BRL, Gaithersburg, MD, USA) according to the manufacturer's instructions. The extracted RNA was denatured at $70{ }^{\circ} \mathrm{C}$ for $10 \mathrm{~min}$, and reverse transcription was performed in reactions containing $5 \mathrm{mM} \mathrm{MgCl}_{2}$, $10 \mathrm{~mm}$ Tris- $\mathrm{HCl}$ ( $\mathrm{pH} 9.0$ ), $50 \mathrm{~mm} \mathrm{KCl}, 0.1 \%$ TritonX100, $1 \mathrm{~mm}$ of each dNTP, $0.5 \mathrm{U}$ of recombinant RNasin ribonuclease inhibitor, $15 \mathrm{U}$ of AMV reverse transcriptase (Promega, Madison, WI, USA) and 500 ng oligo (dT) primer at $42{ }^{\circ} \mathrm{C}$ for $1 \mathrm{~h}$. Using this cDNA as a template, locus-specific PCRs were performed using Amplitaq Gold DNA polymerase (Applied Biosystems, Foster City, CA, USA) and locus-specific primers (Lee et al., 2005). Thermal cycling was performed in a GeneAmp PCR system 2700 (Applied Biosystems). Reaction profiles included a 10-min denaturation step at $94{ }^{\circ} \mathrm{C}$ followed by 38 cycles, each consisting of $30 \mathrm{~s}$ of denaturation at $94{ }^{\circ} \mathrm{C}, 30 \mathrm{~s}$ of appropriate annealing temperature between 60 and $64{ }^{\circ} \mathrm{C}, 1 \mathrm{~min}$ of extension at $72{ }^{\circ} \mathrm{C}$, and then a final 10 min extension step at $72{ }^{\circ} \mathrm{C}$. Cloning was carried out using pGEM-T Easy plasmid vector (Promega) and multiple clones were sequenced. For each SLA gene, more than three clones were sequenced and analysed. A second RT-PCR and cloning reaction was performed for each SLA gene to confirm the polymorphic sequences. New SLA alleles were subjected to allele name assignment by the International Society for Animal Genetics (ISAG) SLA Nomenclature Committee based on a combination of phylogeny and sequence comparison (Smith et al., 2005a,b).

A total of 23 alleles were identified at eight SLA loci in the three Korean native pig boars (Table 1). Their nucleotide and amino acid sequences have been deduced and submitted to the GenBank database. The detailed information of these alleles, including the phylogenetic analyses with all other known SLA alleles and multiple sequence alignments, is available at the SLA section of the Immuno Polymorphism Database (IPD-MHC) website (http://www.ebi.ac.uk/ipd/mhc/sla/) (Ellis et al., 2006). Comparison with previously identified SLA alleles indicated that nine of them were novel and therefore assigned tentative allele names by the ISAG SLA Nomenclature Committee. In addition, five alleles were found to be 
Table 1. The swine leucocyte antigen (SLA) class I and class II alleles identified in three Korean native pigs

\begin{tabular}{|c|c|c|c|}
\hline Locus/Pig ID & 1205 & 1159 & 1119 \\
\hline SLA-1 & $\begin{array}{l}\text { W11jh01 } \\
0801^{\mathrm{b}}\end{array}$ & $\mathrm{w} 11 \mathrm{jh} 01^{\mathrm{a}}$ & w11jh02 \\
\hline SLA-3 & $\begin{array}{l}0303 \\
0701\end{array}$ & 0303 & $05 j \mathrm{jh01^{ \textrm {a } }}$ \\
\hline SLA-2 & $\begin{array}{l}\text { jh01 } \\
0502^{\mathrm{b}}\end{array}$ & $\mathrm{jh} 01^{\mathrm{a}}$ & $\mathrm{jh} 02^{\mathrm{a}}$ \\
\hline SLA-6 & $\begin{array}{l}\text { w04jh01 } \\
0101\end{array}$ & w04jh01 ${ }^{a}$ & 0102 \\
\hline DRA & $\begin{array}{l}020202^{b} \\
0201 j h 01^{a}\end{array}$ & 020202 & 010101 \\
\hline DRB1 & $\begin{array}{l}1101^{\mathrm{b}} \\
10 \mathrm{jh} 01^{\mathrm{a}}\end{array}$ & 1101 & 0101 \\
\hline DQA & $\begin{array}{l}\text { 02jh01 } \\
0101\end{array}$ & $02 \mathrm{jh} 01^{\mathrm{a}}$ & 0101 \\
\hline DQB1 & $\begin{array}{l}0503^{b} \\
0601\end{array}$ & 0503 & 0101 \\
\hline
\end{tabular}

${ }^{\mathrm{a}}$ New alleles; ${ }^{\mathrm{b}}$ Confirmed alleles.

The nucleotide sequence data reported here have been submitted to the DDBJ/EMBL/GenBank nucleotide databases under the accession numbers DQ883208-DQ883227 and EF589959-EF589961.

identical to the tentative alleles previously recognized (SLA-1*w08sz01, SLA-2*05sz01, SLA-DRA*0202 mm16, SLA-DRB1*w11an01 and SLA-DQB1*05an01) (Smith et al., 2005a,b). These alleles were therefore renamed as confirmed alleles SLA-1*0801, SLA-2*0502, SLADRA*020202, SLA-DRB1*1101 and SLA-DQB1*0503, respectively. Furthermore, results suggested that boar 1159 was homozygous for a novel SLA haplotype (Hp-56.30) while boar 1119 was homozygous for another novel class I haplotype and the Hp- 0.1 class II haplotype which was identical to the H01 haplotype found in the Large White pig breed (Smith et al., 2005b). Therefore, the assigned SLA haplotype of animal 1119 is Hp-59.1. This suggested some common genetic background between the European breeds and the Korean native pigs. In order to improve the production ability of native pigs in Korea, it was also possible to crossbreed with the highly productive pigs such as Large White. Comparison of the alleles also suggested that boar 1205 was heterozygous for SLA haplotypes Hp-7.23 and Hp-56.30 (Smith et al., 2005a). Typing of the offspring is necessary in the future to show the inheritance and segregation of the alleles as haplotypes and therefore provide further confirmation for haplotype assignments in these three pigs. In conclusion, these new SLA alleles and haplotypes contribute to the understanding of polymorphism of these genes and give us better tools to study the role of SLA antigens in xenotransplantation and in important agricultural traits such as disease resistance.

\section{Acknowledgements}

This work was supported by the On-Site Cooperative Agriculture Research Project (project No. 20070401080056), Rural Development Administration, South Korea, and by the research project on the Production of Bio-organs (project no. 200702078) from Biogreen 21 program, Rural Development Administration, South Korea. This work was also partly supported by grant no. R11-2002-100-00000-0 from the ERC program of the Korea Science and Engineering Foundation.

\section{References}

Ellis, S.A., Bontrop, R.E., Antczak, D.F., Ballingall, K., Davies, C.J., Kaufman, J. et al. (2006) ISAG/IUIS-VIC Comparative MHC Nomenclature Committee report, 2005. Immunogenetics, 57, 953.

Gautschi, C. \& Gaillard. C. (1990) Influence of major histocompatibility complex on reproduction and production traits in swine. Animal Genetics, 21, 161.

Lee, J.H., Simond, D., Hawthorne, W.J., Walters, S.N., Patel, A.T., Smith, D.M., O'Connell, P.J. \& Moran, C. (2005)

Characterization of the swine major histocompatibility complex alleles at eight loci in Western pigs. Xenotransplantation, 12, 303.

Mallard, B.A., Kennedy, B.W. \& Wilkie, B.N. (1991) The effect of swine leukocyte antigen haplotype on birth and weaning weights in miniature pigs and the role of statistical analysis in this estimation. Journal of Animal Science, 69, 559.

Ramachandran, S., Jaramillo, A., Xu, X.C., McKane, B.W., Chapman, W.C. \& Mohanakumar, T. (2004) Human immune responses to porcine endogenous retrovirus-derived peptides presented naturally in the context of porcine and human major histocompatibility complex class I molecules: implications in xenotransplantation of porcine organs. Transplantation, 77, 1580.

Smith, D.M., Lunney, J.K., Martens, G.W., Ando, A., Lee, J.H., Ho, C.S., Schook, L., Renard, C. \& Chardon, P. (2005a) Nomenclature for factors of the SLA class-I system, 2004. Tissue Antigens, 65, 136.

Smith, D.M., Lunney, J.K., Ho, C.S., Martens, G.W., Ando, A., Lee, J.H., Schook, L., Renard, C. \& Chardon, P. (2005b) Nomenclature for factors of the swine leukocyte antigen class II system. Tissue Antigens, 66, 623.

Xu, X.C., Naziruddin, B., Sasaki, H., Smith, D.M. \& Mohanakumar, T. (1999) Allele-specific and peptide-dependent recognition of swine leukocyte antigen class I by human cytotoxic T-cell clones. Transplantation, 68, 473. 